\title{
Noise evaluation of a point autofocus surface topography measuring instrument
}

\author{
Giacomo Maculotti ${ }^{1}$, Xiaobing Feng ${ }^{2}$, Maurizio Galetto ${ }^{1}$, Richard Leach $^{2}$ \\ ${ }^{1}$ Department of Management and Production Engineering, Politecnico di Torino, Italy \\ ${ }^{2}$ Manufacturing Metrology Team, Faculty of Engineering, University of Nottingham, UK
}

\begin{abstract}
In this work, the measurement noise of a point autofocus surface topography measuring instrument is evaluated, as the first step towards establishing a route to traceability for this type of instrument. The evaluation is based on the determination of the metrological characteristics for noise as outlined in draft ISO specification standards by using a calibrated optical flat. The static noise and repeatability of the autofocus sensor are evaluated. The influence of environmental disturbances on the measured surface topography and the built-in software to compensate for such influences are also investigated. The instrument was found to have a measurement noise of approximately $2 \mathrm{~nm}$ or, when expressed with the measurement bandwidth, $0.4 \mathrm{~nm} / \sqrt{\mathrm{Hz}}$ for a single-point measurement.
\end{abstract}

Keywords: metrological characteristics, measurement noise, point autofocus instrument, static noise, autofocus repeatability, noise bandwidth specification, environment sensitivity

\section{INTRODUCTION}

Surface texture affects the functional properties of engineered surfaces, such as surface energy (e.g. capillarity, wetting, adhesion), optical (e.g. reflectivity, absorption, diffraction) and thermal (e.g. conduction and radiation heat transfer mechanism) characteristics. Surfaces can also be engineered for bio-compatibility, mechanical fatigue, hydrodynamic and tribological performance [1,2]. Optimisation of surface texture is required in various applications to improve the performance of products. For example, automobile engine parts (cylinder liners, piston pins and oil rings) with optimised surface texture have been demonstrated to improve fuel efficiency and service life [3-5]; when coating cutting tools, surface texture is critical in affecting wettability and interactions between the coating and substrate, which in turn determines the wear behaviour and life of the coated tools [6]. Texturing the surface of medical implants is used to improve osseointegration at the implant-bone interface [7]; Fresnel lenses utilise surface texturing to achieve focusing power at significantly lower thickness and mass; and sub-wavelength optical structures have been used to produce anti-reflective surfaces, polarizers and beam splitters [8].

To optimise the performance of the above-mentioned products, it is important to have confidence in the accuracy of surface topography measurements, which can be ensured by establishing traceability. This can be achieved by evaluating the metrological characteristics (MCs) of the surface topography measuring instrument using calibrated artefacts [9]. The MCs for a contact stylus instrument, a coherence scanning interferometer and an imaging confocal microscope have been investigated [1012], and there has been some research on characterising noise for a focus variation instrument [13]. In this work, we evaluate the MCs for a point autofocus instrument (PAI) for the measurement of areal surface topography. The PAI is an optical measuring instrument that automatically focuses a laser beam to a single point on the surface and raster scans an area of interest [14]. PAIs are often used to measure optics, cutting tools, and micro-gears. The general characteristics and MCs of PAIs are 
introduced in the specification standard ISO 25178-605 [15]. However, there is currently no established method for determining the MCs specifically for PAI. As the first step towards establishing traceability of this type of instrument, the measurement noise, static noise and autofocus repeatability of a commercial instrument (Mitaka Kohki MLP-3SP) are evaluated. The remaining MCs will be addressed in future papers.

The rest of this paper is structured as follows: section 2 provides an introduction to the measurement mechanism of PAIs; section 3 describes the methodology used to evaluate the MCs as defined in ISO 25178-605; section 4 presents the results; and section 5 concludes the findings.

\section{INSTRUMENTATION}

A PAI is a non-contact, optical areal topography measuring instrument, which consists of a laser source, a microscope objective, an autofocus mechanism and a precision moving stage [14,15]. The laser beam is focused onto the surface so that the focal spot defines a height of a single point on the surface. The PAI used for this work is a commercial instrument (MLP-3SP) hosted in the laboratory of the Manufacturing Metrology Team of the University of Nottingham. In this instrument, autofocus is achieved using the beam-offset method [14]: the incident beam passes through one side of the objective lens and is focused onto a point on the sample surface; the reflected beam passes through the opposite side of the objective lens and is received by the autofocus sensor. The detected laser spot displacement is used as the feedback signal in the autofocus mechanism to adjust the position of the objective lens. When the objective lens is at an in-focus position, surface height is computed as the sum of the position of the vertical $z$-axis and the autofocus (AF) axis [14]. Movement along the $x, y$ and $z$ axes is determined by linear scales with a nominal resolution of $10 \mathrm{~nm}$, while movement along the AF axis is determined by a linear scale with a nominal resolution of $1 \mathrm{~nm}$. The instrument features a chamber that shields the measurement from external environmental disturbances.

\section{METHODOLOGY}

The MCs of an optical measuring instrument are influenced by several factors, such as environmental, mechanical and electrical noise, optical aberrations and mathematical algorithms. To assess the contribution of each individual factor would be time consuming and often unnecessary for the end user. Thus, an input-output model has been introduced [14] to account for the influence factors using the MCs introduced in the draft standard ISO/DIS 25178-600 [16], and defined as characteristics of the measuring equipment, which may influence the result of measurement, may require calibration and have an immediate contribution to measurement uncertainty. Eight MCs are included in the draft standard specification: measurement noise, flatness deviation, amplification coefficient, linearity deviation, $x-y$ perpendicularity deviation, topographic spatial resolution, topography fidelity and maximum measurable local slope [9].

Measurement noise $N_{M}$ is defined in ISO/FDIS 25178-600 as the noise added to the output signal occurring during the normal use of the instrument [16]. Measurement noise is a dynamic phenomenon, which is affected by the motion of the drive unit as well as instrument internal noise and environmental disturbances. Determination of $N_{M}$ is achieved through areal surface topography measurement of a calibrated optical flat artefact. Furthermore, static noise and autofocus repeatability are investigated in order to separate the contribution of the drive unit and that of environmental disturbances. All 
measurements were performed in a temperature-controlled laboratory environment $\left(20{ }^{\circ} \mathrm{C} \pm 0.5{ }^{\circ} \mathrm{C}\right)$, unless otherwise stated.

\subsection{Measurement noise}

Two methods have been proposed in the literature [10] to evaluate $N_{M}$ : the subtraction method and the averaging method. Both methods require repeated measurement of a calibrated optical flat and describe the $N_{M}$ in terms of the root mean square height of the surface $S q$. The subtraction method evaluates $N_{M}$ by subtracting consecutively measured surface topographies to try to remove the effect of the finite topography of the flat. As the subtraction combines the variances of two identical probability distributions that each characterise the noise of the instrument, $N_{M}$ can be estimated using the topography resulting from the subtraction of the two, divided by the square root of two:

$$
S_{\text {qnoise }}=\frac{S_{q}}{\sqrt{2}}
$$

The averaging method is based on the assumption that the noise contribution to $S q$ decreases when averaging multiple measurements, i.e. noise is statistically stationary, and that the measured surface topography can be considered as made up of the "true" topography and the noise contribution. With repeated measurements of the same surface area, measurement noise can be estimated by:

$$
S_{\text {qnoise }}=\sqrt{\frac{S_{q}{ }^{2}-S_{q n}{ }^{2}}{1-1 / n}}
$$

where $n$ is the number of averaged topographies and $S_{q n}$ is the root mean square height of the averaged topography [17]. Furthermore, the measurement noise uncertainty contribution (following [10]) propagates with a normal distribution with null expectation and a variance equal to the measurement noise squared, and can be computed accordingly:

$$
u_{\text {noise }}=N_{M}=S_{\text {qnoise }} .
$$

In this work, $N_{M}$ is evaluated over fifteen repeated measurements of an optical flat from a set of artefacts calibrated by the National Physical Laboratory, UK (NPL-BNT 019) [18], with a nominal $S z$ value of $4 \mathrm{~nm}$, with an expanded uncertainty of $10 \mathrm{~nm}$ (note that this specification is from the certificate). The measurement settings are shown in Table 1. The choice of the scanning pitch and stepping pitch results from a trade-off between lateral resolution and measurement duration [19], where the highest lateral resolution is used in the scanning direction while a larger stepping pitch is selected to

\begin{tabular}{|c|c|c|}
\hline \multicolumn{2}{|c|}{ Measured area } & $100 \mu \mathrm{m} \times 100 \mu \mathrm{m}$ \\
\hline \multicolumn{2}{|c|}{ Scanning pitch } & $0.1 \mu \mathrm{m}$ \\
\hline \multicolumn{2}{|c|}{ Stepping pitch } & $1 \mu \mathrm{m}$ \\
\hline Objective & $\begin{array}{c}\text { Magnification } \\
\text { Numerical aperture }\end{array}$ & $\begin{array}{c}100 \times \\
0.8\end{array}$ \\
\hline
\end{tabular}
reduce the measurement duration to approximately one hour.

Table 1 Measurement settings for $N_{M}$ assessment 
Before applying the evaluation methodologies, post-processing of the acquired topographies is performed using the commercial software MountainsMap ${ }^{\circledR}$, which includes:

- levelling the surface by subtraction of the least-squares mean plane; and

- removing outliers, mostly due to contamination, by applying a threshold of $0.5 \%$ and $99.5 \%$ of the material ratio.

Measurement noise is a common performance specification cited by instrument manufacturers, as it aids in quantifying measurement repeatability and vertical resolution. In particular, the definition of vertical resolution is not consistent among various instrument manufacturers and may cause difficulty when comparing different instruments [20]. Although $N_{M}$ is an effective alternative to quantify the minimum detectable vertical distance, without the need of either defining the vertical resolution or designing a dedicated material measure, it can be affected by the temporal bandwidth of the measurement. For example, various types of environmental disturbance can introduce noise in different bandwidths; and noise can be reduced by averaging signals over a longer duration [20]. Therefore, to create a common reference frame for describing measurement noise, it is necessary to describe $N_{M}$ along with the associated measurement bandwidth, expressing it in terms of noise equivalent height, in $\mathrm{nm}$, divided by the square root of the data acquisition rate, in height points per second.

\subsection{Static noise}

Static noise evaluation complements $N_{M}$ when describing the noise affecting the instrument. The investigated PAI, despite being an optical instrument, is not an imaging system; on the other hand, due to its working principle, it can be treated as an optical equivalent of a contact stylus. Therefore, this work evaluates the static noise on the basis of ISO 25178-701 [21]: the laser beam is focused on a calibrated optical flat (from NPL-BNT 019) and fluctuations in the height of the measured point are recorded. Static noise is then computed as the standard deviation of the recorded surface height signal; and describes the repeatability in the vertical direction, which is affected by both the $z$ and AF axes.

The height of a single point (focal spot) is recorded for a period of fifteen minutes. As the instrument is not in measurement mode during this investigation, the fluctuations in surface height can only be displayed on the instrument screen, but not saved as a file. Therefore, surface height information is retrieved from the video recording of the monitor screen at twenty-four frames per second, and then sampling the signal at $2 \mathrm{~Hz}$ to avoid under-sampling.

\subsection{Autofocus sensor repeatability}

The autofocus repeatability $R_{\mathrm{AF}}$ is a characteristic introduced in ISO 25178-605 [15] specific to the working mechanism of PAI. $R_{\mathrm{AF}}$ aims at characterising the AF axis while excluding other influence factors, such as the $z$ axis fluctuations and environmental disturbances. $R_{\mathrm{AF}}$ is determined using an internal instrument software function, which repeatedly focuses the laser beam onto the same point and records the position of the autofocus sensor. In this work, 1500 measurements were repeated with a sampling interval of $1.5 \mathrm{~s}$. The measurement procedure was then repeated five times. $R_{\mathrm{AF}}$ is determined as the standard deviation of the recorded AF axis readings. 


\section{RESULTS AND DISCUSSION}

\subsection{Environmental effects}

Before evaluating noise, an issue affecting the measured topography needed to be addressed. When measuring an optical flat in a temperature-controlled environment, noticeable deviation from the ideal geometry is present on the measured topography, as shown in Figure 1(a). The topography consists of an overall waviness superimposed on the nominal topography of the optical flat. The deviation indicates a drift in surface height over time (an approximately one-hour period in this case). A built-in function is available in the instrument software to compensate for the drift, and when enabled, significantly reduces the previously observed deviation, as shown in Figure 1(b). The compensation function regularly corrects the topography by actively monitoring the magnitude of drift in the height of a pre-defined point and subtracting this magnitude from the measured profiles. The frequency of applying the correction can be specified, which ranges from applying it to every scanned profile, to a set number of profiles. With the increased correction frequency, drift is better compensated, however, measurement duration is increased. As this function is recommended by the instrument manufacturer for areal measurement, it was enabled for all evaluations of measurement noise in this study. To minimise the influence of drift, compensation was set to be performed as often as possible (i.e. on every scanned profile). However, due to time delays in monitoring the drift and noise in the AF axis, errors are inevitably introduced during compensation, resulting in height changes between scanned profiles as shown in Figure 1(b). 
(a)
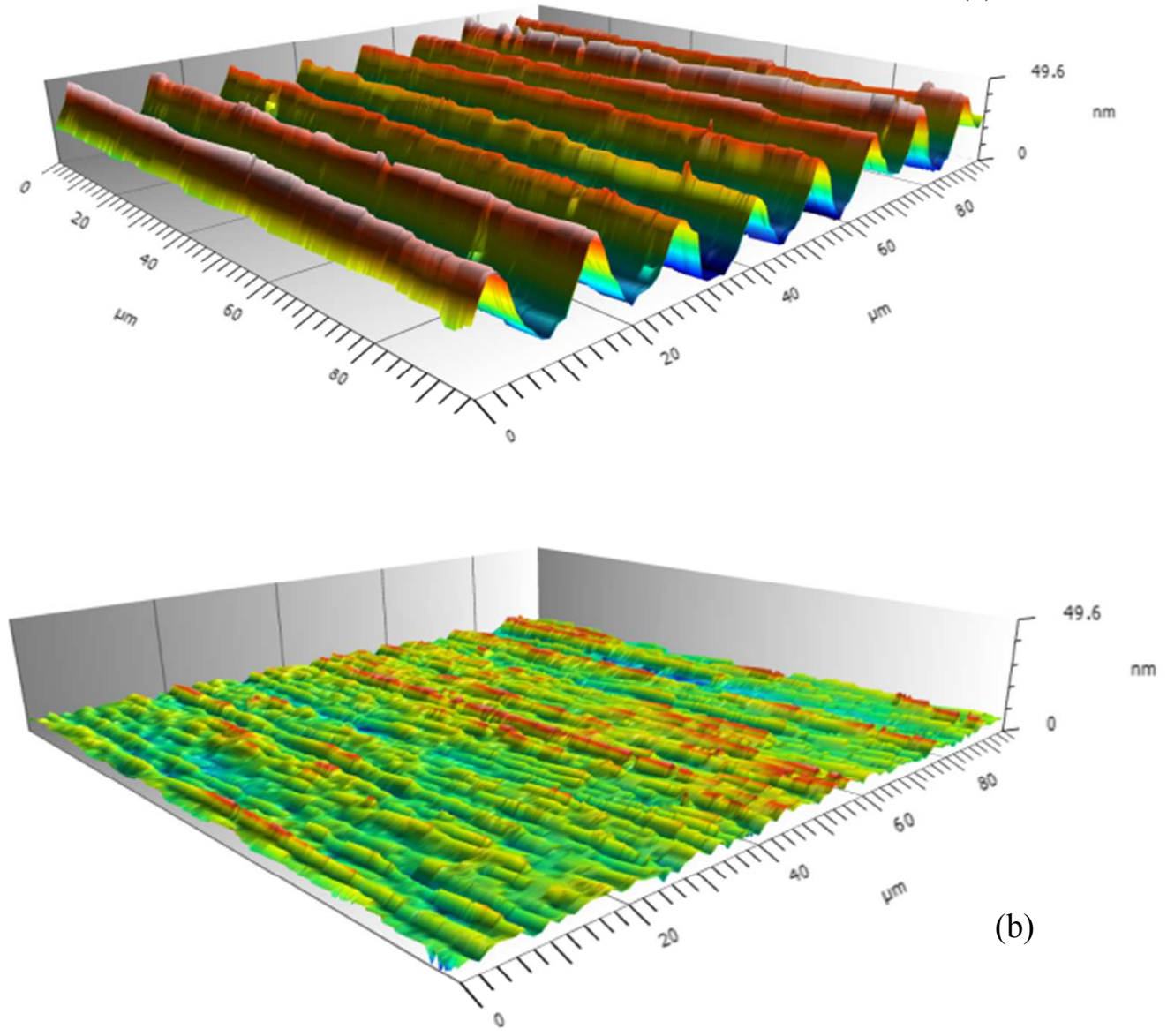

Figure 1 Example of measured surface topography of an optical flat (a) without application of drift compensation function and (b) with application of the drift compensation function is applied.

Even though drift can be largely compensated by the built-in function, it is important to first understand the cause of the drift. In this section, the nature of the drift is analysed, and the potential cause of the drift is explored. Given the periodic behaviour of the deviation and the nature of the temperature control in the laboratory, it was suspected that the intermittent switching on of the air conditioning system had caused the deviation. Therefore, an investigation was conducted to assess whether a correlation between the periodic deviation and temperature fluctuations can be found. The investigation also aims to determine the effectiveness of the built-in drift compensation function.

The instrument is modelled as a black box, which receives as input the nominal topography combined with the noise signal, and outputs the measured surface topography. In particular, the noise can be considered as made up of several contributions, where that due to the temperature fluctuations is the main focus of this section. To verify the correlation between the temperature fluctuations and the periodic deviation in the measured topography, the temperature inside the instrument measurement chamber during the measurement period is recorded by a resistance temperature detector PT-1000 twowire probe with a nominal accuracy of $\pm 0.15^{\circ} \mathrm{C}$. 
Linear systems theory guarantees that the input and the output signals have at least the same harmonic content in their spectra, i.e. a harmonic at the same frequency; therefore, a common harmonic is searched between the recorded temperature and the deviation in the resulting surface topography. The frequency spectrum of the recorded temperature is computed using a fast Fourier transform (FFT) algorithm. The deviation in the topography is represented by the mean surface profile along the stepping direction, as shown in Figure 2.

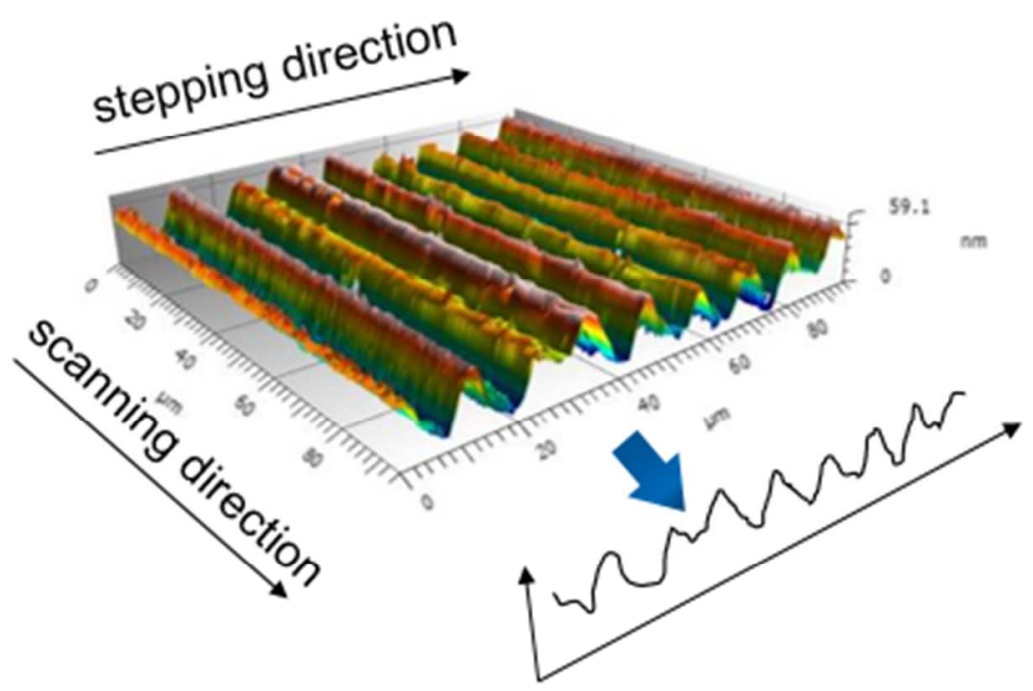

Figure 2 Mean surface profile extraction along the stepping direction.

To consistently compare the frequency spectra of the temperature and topographical signals, the spatial sampling frequency of the mean surface profile $\left(1 \mu \mathrm{m}^{-1}\right)$ along the stepping direction needs to be converted to a temporal frequency $f_{T}$ considering an overall measurement duration of fifty-five minutes:

$$
f_{T}=\frac{101 \text { lines }}{55 \mathrm{~min} \times 60 \mathrm{~s} / \mathrm{min}}=0.0306 \mathrm{~Hz} \text {. }
$$

Fifteen repeated measurements of the same area of the flat were carried out with the setup shown in Table 1; temperature inside the instrument chamber was recorded at a sampling frequency of $1 \mathrm{~Hz}$.

Figure 3 shows the recorded chamber temperature during the fifteen repeated areal measurements. It can be seen that the first measurement was associated with a steep increase in temperature in the measurement chamber, which is likely due to instrument warm-up. 


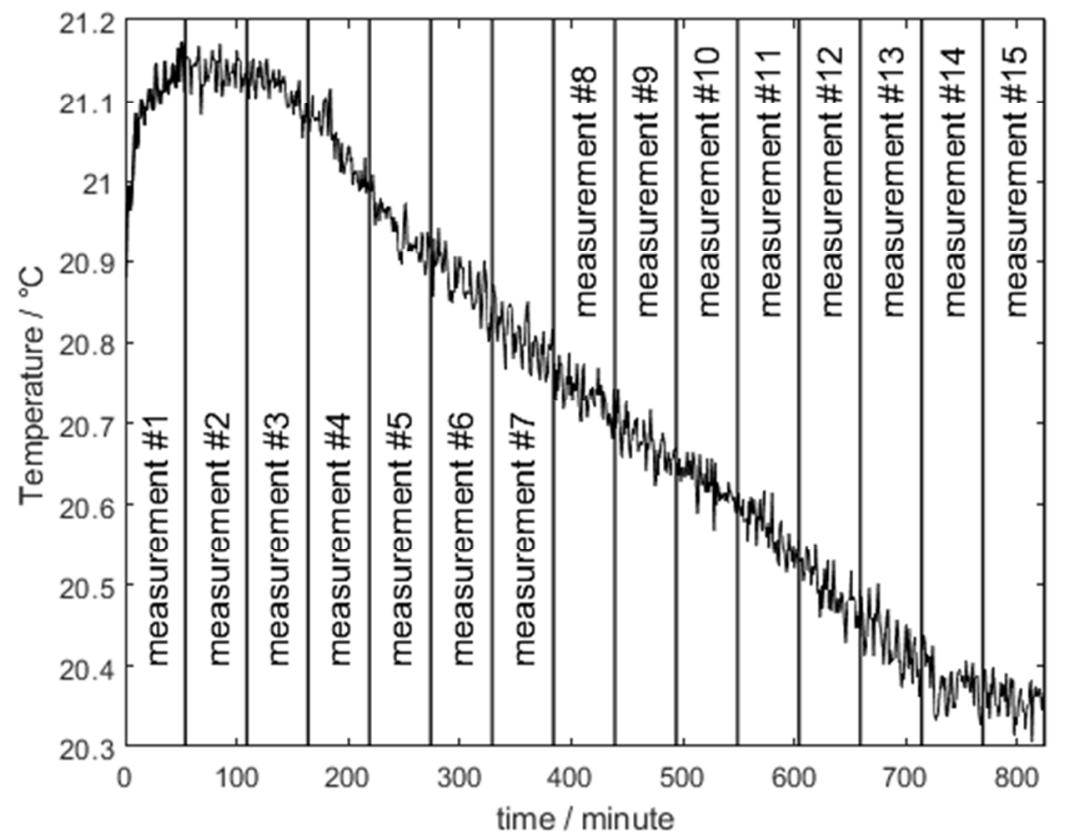

Figure 3 In-chamber temperature during fifteen repeated areal topography measurements, with active room temperature control. Vertical lines indicate the end of each measurement.

Figure 4 shows the frequency spectra of the in-chamber temperature and the mean surface profile for one of the measurements. The presence of a common harmonic indicates a potential correlation between the two signals. The harmonic frequencies found in all fifteen measurements were analysed, and the results are shown in Figure 5. The overlap of the expanded uncertainty intervals, with coverage factor $k$ equal to 2, between the in-chamber temperature and the topographical deviations suggests a degree of correlation. Therefore, temperature fluctuations in the laboratory are believed to be the cause of the waviness added to the measured topography.

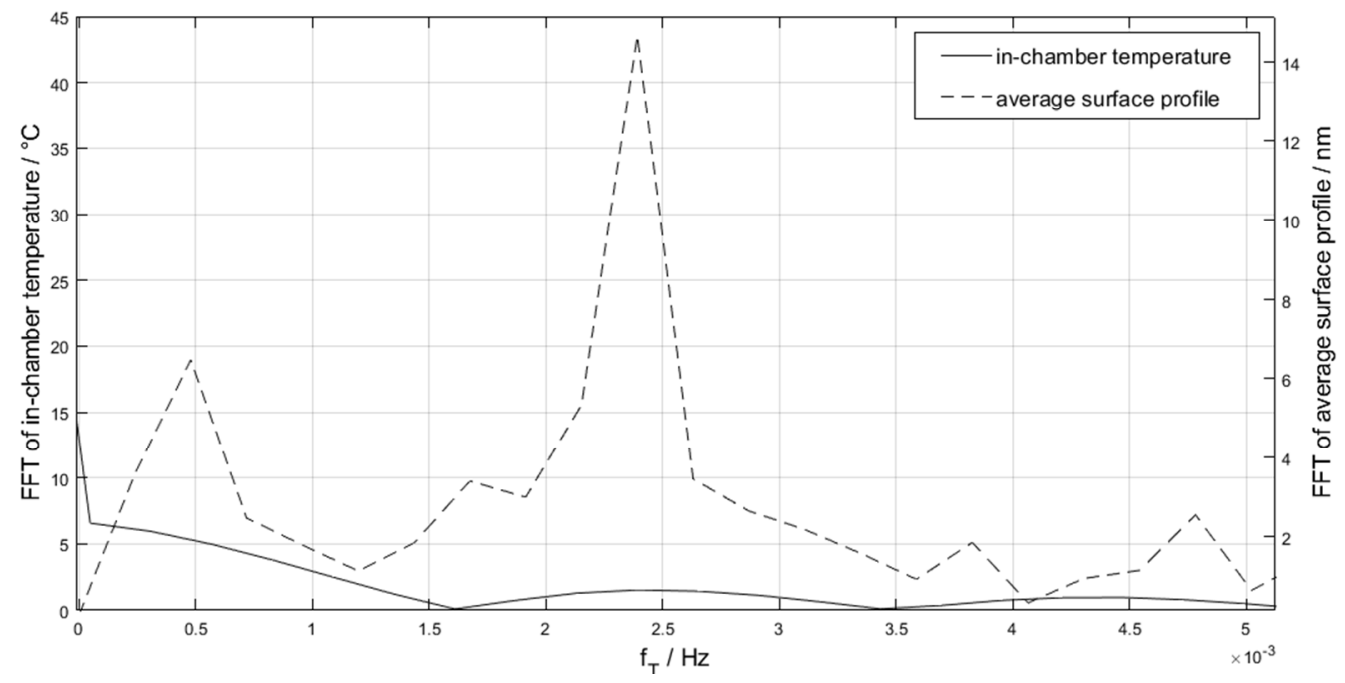

Figure 4 Frequency spectra of the mean surface profile and in-chamber temperature. 


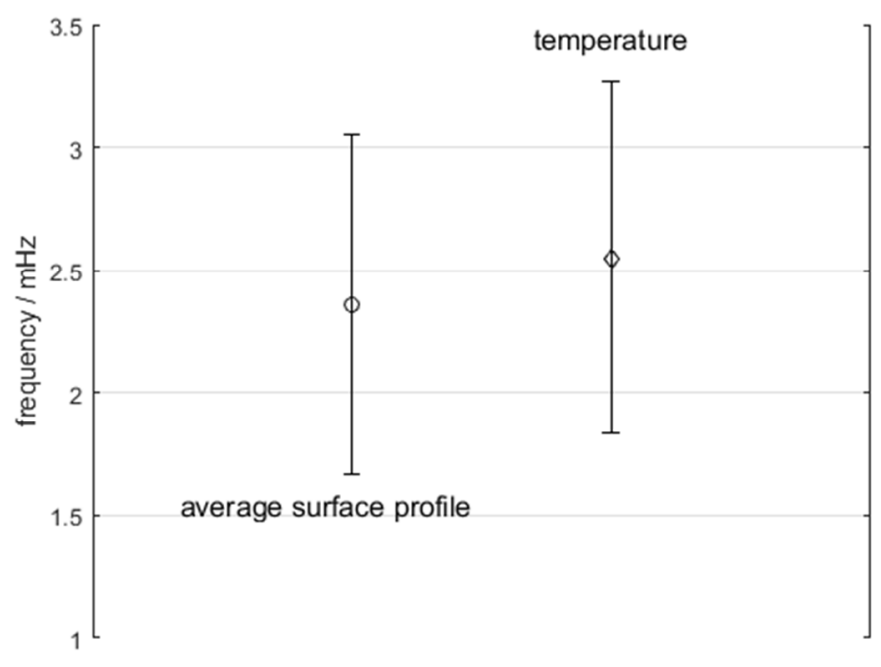

Figure 5 Frequency of the principal harmonics of the mean surface profile and in-chamber temperature. Error bands show expanded uncertainty $(k=2)$.

Figure 6 shows that after the built-in drift compensation function is applied, a principal harmonic is no longer present in the frequency spectra of the mean surface profile and the magnitude decreases significantly, indicating that the drift induced by temperature fluctuation is effectively compensated.

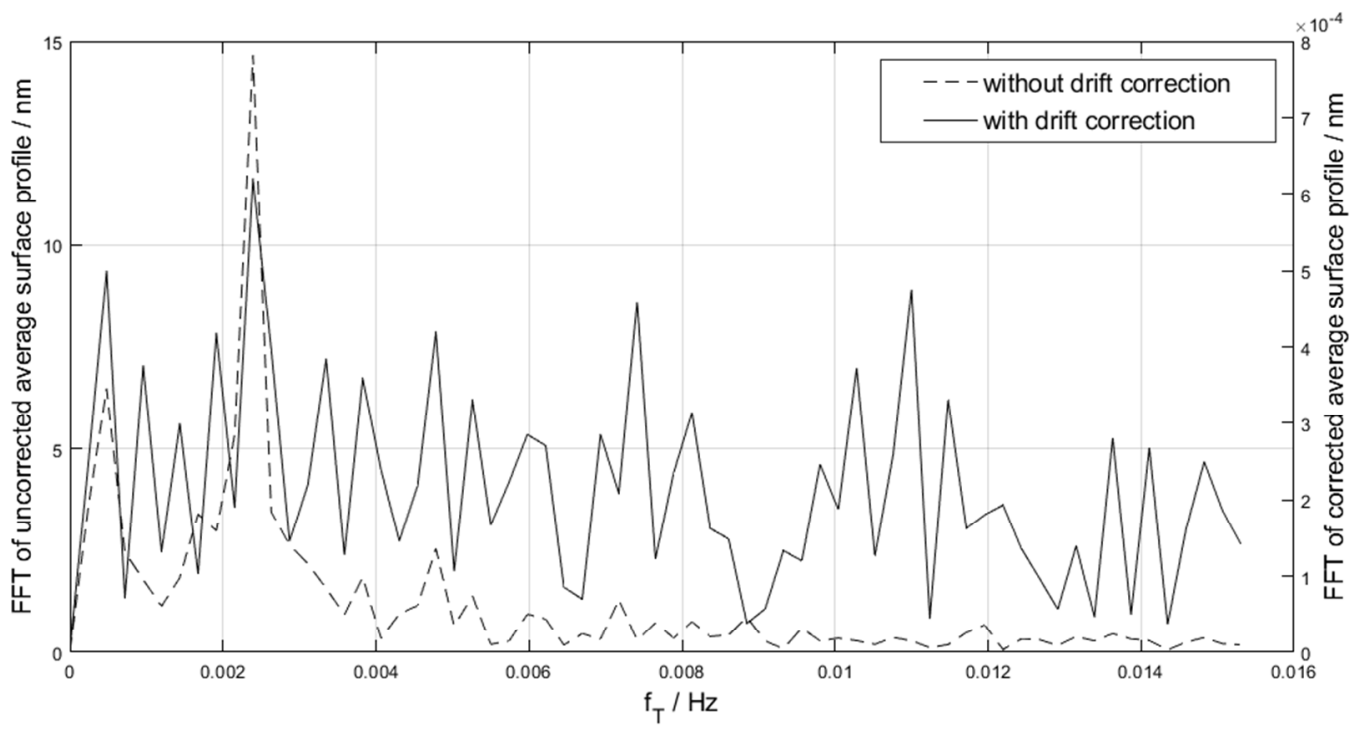

Figure 6 Frequency spectra of the mean surface profile with and without drift compensation.

To further demonstrate that temperature variation is the main cause of the drift in surface height, Figure 7 (a) shows an example profile along with the corresponding chamber temperature during the measurement period. A good correlation between chamber temperature and surface height can be observed in Figure 7(b). 
(a)

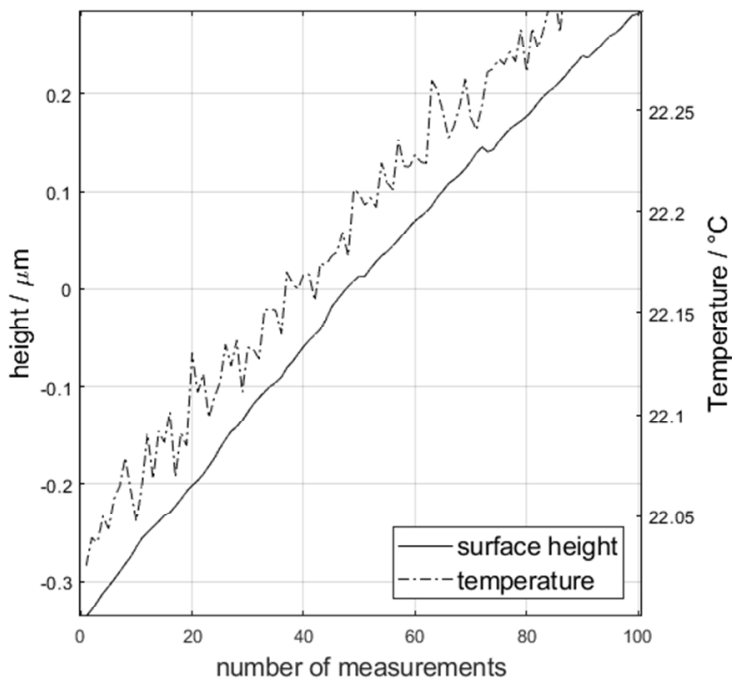

(b)

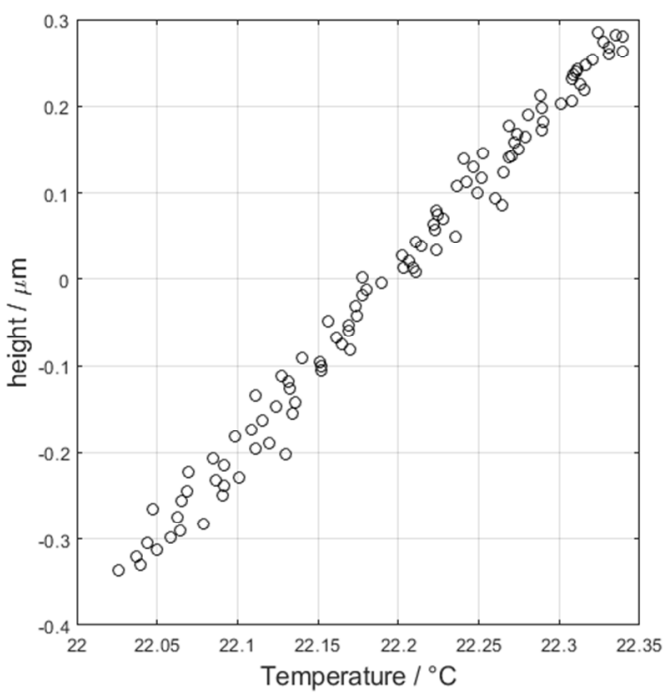

Figure 7 (a) A surface profile extracted from an areal measurement and chamber temperature during the measurement; temperature control was disabled. (b) Correlation between surface profile height and chamber temperature.

The influence of temperature on measured surface height, as indicated in Figure 7, is a common source of measurement error, and has been reported with contact probes [22], displacement transducers (e.g. strain gauges, piezoelectric, variable resistance and variable inductance displacement transducers) $[23,24]$, nano-scale sensors, inductive probes, capacitive probes and laser interferometer [25].

\subsection{Measurement noise}

Measurement noise was evaluated using both the subtraction and the averaging methods introduced elsewhere [10]. The built-in drift compensation function was enabled to minimise the influence of environmental temperature fluctuations. Examples of the topographies resulting from the application of the two methods are shown in Figure 8(a) and (b). The resulting measurement noise values are shown in Table 2, calculated with equations (1) and (2). 
(a)

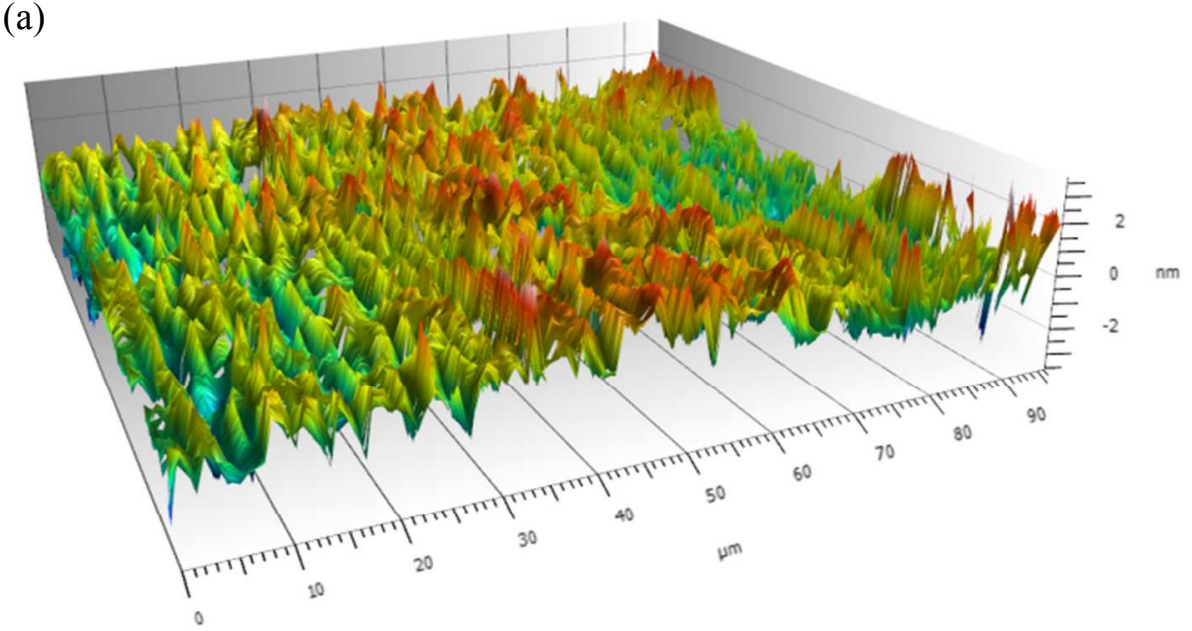

(b)

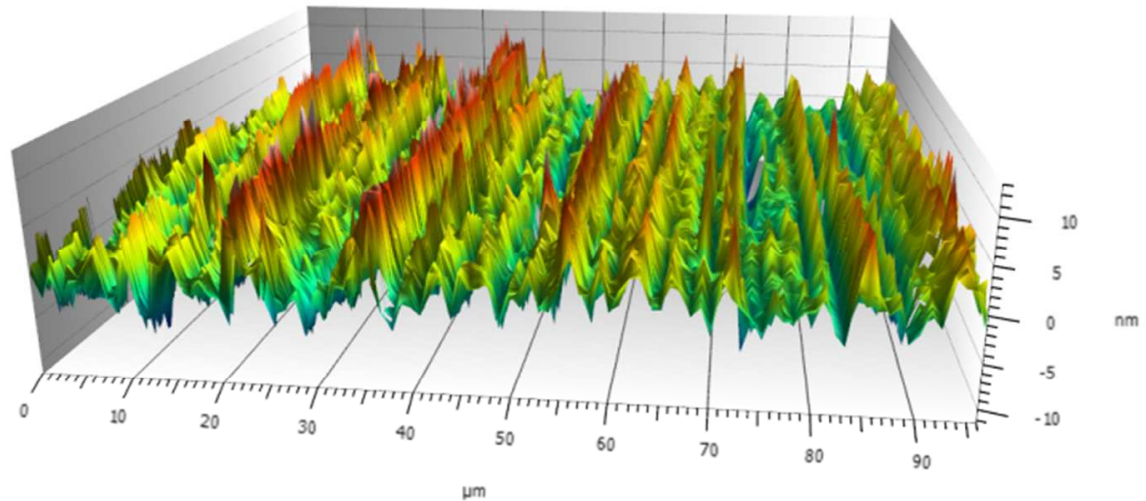

Figure 8 Surface topographies used to determine measurement noise: (a) the mean of fifteen repeatedly measured topographies, and (b) the difference between two consecutively measured topographies after subtraction.

It was found that $N_{M}$ values stabilise at approximately $2 \mathrm{~nm}$. Given that the least discernible digit of the AF sensor is $1 \mathrm{~nm}, N_{M}$ and its contribution to uncertainty were determined to be $2 \mathrm{~nm}$, accordingly to equation (3).

With both the subtraction and the averaging methods, the number of repeated measurements required to reach a stable value of $N_{M}$ cannot be easily determined and depends on the instrument being evaluated. In the case of the PAI under evaluation, five repeated measurements were found to be sufficient, which is less than the fifteen measurements performed in this work and less than the number suggested in literature for other instruments [10].

Table 2 Measurement noise $N_{M}$ computed using both averaging and subtraction methods.

\begin{tabular}{ccccccccccccccc}
\hline $\boldsymbol{n}$ & $\mathbf{2}$ & $\mathbf{3}$ & $\mathbf{4}$ & $\mathbf{5}$ & $\mathbf{6}$ & $\mathbf{7}$ & $\mathbf{8}$ & $\mathbf{9}$ & $\mathbf{1 0}$ & $\mathbf{1 1}$ & $\mathbf{1 2}$ & $\mathbf{1 3}$ & $\mathbf{1 4}$ & $\mathbf{1 5}$ \\
\hline $\begin{array}{c}\boldsymbol{N}_{\boldsymbol{M}} / \mathbf{n m} \\
\text { averaging method }\end{array}$ & 1.73 & 2.06 & 1.90 & 2.04 & 2.13 & 2.14 & 2.10 & 2.11 & 2.10 & 2.08 & 2.09 & 2.07 & 2.08 & 2.07 \\
\hline $\begin{array}{c}\boldsymbol{N}_{\boldsymbol{M}} / \mathbf{n m} \\
\text { subtraction } \\
\mathbf{m e t h o d}\end{array}$ & 2.10 & 1.89 & 2.02 & 1.84 & 2.27 & 2.33 & 2.16 & 2.10 & 1.78 & 2.09 & 2.05 & 1.91 & 2.00 & 2.21 \\
\hline
\end{tabular}

To further evaluate the effectiveness of the built-in drift compensation function, $N_{M}$ values determined both with and without applying the drift compensation function were compared. It was found that $N_{M}$ 
can be as large as $20 \mathrm{~nm}$, in the worst case obtained using the averaging method. Stabilisation of the $N_{M}$ value also became more difficult to achieve, as temperature fluctuations were different during each repeated measurement. In contrast, when drift compensation was applied, $N_{M}$ was reduced to $2 \mathrm{~nm}$ and stabilisation of noise values was achieved within five repeated measurements every time, indicating stable behaviour. Measurements were also performed on a shop floor without any temperature control measures; and similar noise values were found, indicating that the drift compensation function was effective in both laboratory and manufacturing environments and that strict temperature control is not necessary for the instrument.

With the spatial sampling settings described in Table 1, a total of $1001 \times 101$ points were measured in approximately fifty-five minutes, resulting in a measurement bandwidth of $30.6 \mathrm{~Hz}$. As a result, the bandwidth specification of measurement noise was determined to be $0.4 \mathrm{~nm} / \sqrt{\mathrm{Hz}}$ for a single-point measurement.

\subsection{Static noise}

Static noise was evaluated to complete the description of the noise affecting the instrument and it excludes any noise in the drive unit involved in raster scanning. The recorded height of a single point on the optical flat is shown in Figure 9 along with the measured temperature during the investigation. The same drift that affected the areal measurement was found in the recorded height signal. Since such drift would have been compensated for in areal measurement, it is reasonable to remove the drift when analysing the static noise of the instrument. Removal of the drift was achieved by applying a high pass filter with a cut-off frequency associated with the fundamental frequency of the in-chamber temperature, which was found to be approximately $10 \mathrm{mHz}$. Static noise, determined as the standard deviation of the residual height, is computed to be $2 \mathrm{~nm}$. Spikes with magnitudes of approximately $10 \mathrm{~nm}$ are observed in Figure 9, which are due to the fluctuations in the $z$ axis position, where the smallest discernible difference in the encoder scale is $10 \mathrm{~nm}$. The spikes were not removed before applying the high pass filter in order to conform to the definition of static noise, which accounts for noise in both the vertical axis and the autofocus sensor.

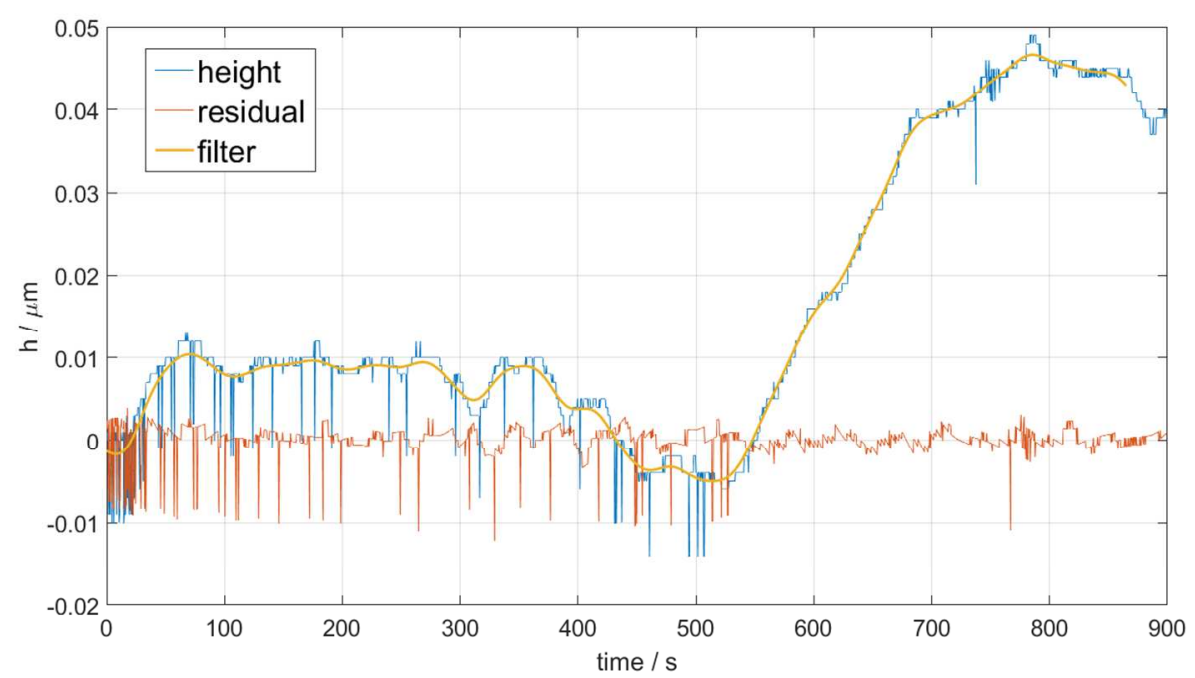

Figure 9 Continuously recorded height of a single point on the optical flat. (Blue: recorded height signal. Orange: residual height signal after Gaussian filtering. Yellow: drift component in the recorded height signal.). 


\subsection{Autofocus sensor repeatability}

Figure 10 shows the repeatedly measured height of a single point on the optical flat. Similar to the observation in section 4.3, drift in the measurement is present in the form of an oscillation caused by the periodical regulation of room temperature in the laboratory. As the definition of $R_{\mathrm{AF}}$ in ISO 25178605 [15] excludes the influence of environmental disturbance it is, therefore, necessary to remove the drift using a high-pass filter with a cut-off frequency of $4 \mathrm{mHz}$, which was assessed to be the fundamental frequency of the in-chamber temperature fluctuation during $R_{\mathrm{AF}}$ evaluation. The resulting autofocus sensor repeatability was found to be $5 \mathrm{~nm}$.

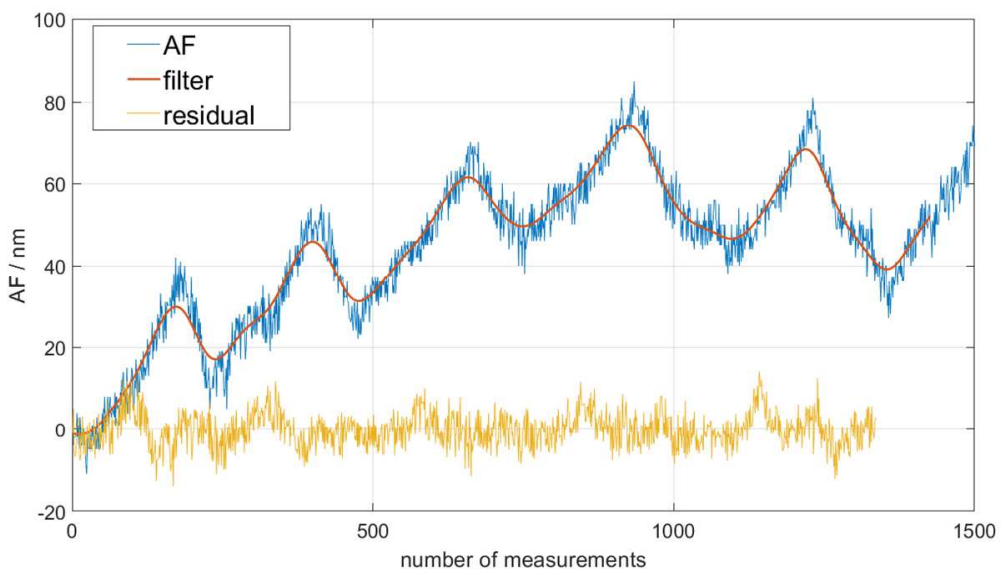

Figure 10 Repeated measurements of the height of a single point on the optical flat, at an interval of $1.5 \mathrm{~s}$. (Blue: recorded autofocus position. Orange: drift component in the AF position. Yellow: residual AF position after Gaussian filtering).

It is worth pointing out that different cut-off frequencies were used when applying the Gaussian filters in section 4.3 and section 4.4. This is because the two measurements were obtained in separate sessions, during which temperature was found to vary in different fashions and frequencies. Therefore, the appropriate cut-off values had to be determined by the actual frequencies of temperature variation during the investigations. Furthermore, Gaussian filtering was only applied when determining static noise and AF repeatability, as the inbuilt drift compensation function is only available during areal measurement.

\section{CONCLUSION}

This work is a first step towards establishing traceability of a PAI and presents methods for evaluating the measurement noise, static noise and autofocus repeatability. The influence of environmental temperature disturbances has been investigated, and the effectiveness of the built-in drift compensation function assessed. When not applying the built-in drift compensation function, a deviation with a periodic nature was observed in the measured surface topography. The deviation was subsequently found to be caused by environmental temperature disturbances, based on analysis of the spectra of the deviation in the topographies and that of the in-chamber temperature. The correlation between inchamber temperature and surface height was also confirmed in the temporal domain. Once the built-in drift compensation function was applied, the periodic deviation was effectively compensated; and measurement noise has been determined to be $2 \mathrm{~nm}$ using both the subtraction method and averaging methods or, when expressed with the measurement bandwidth, $0.4 \mathrm{~nm} / \sqrt{\mathrm{Hz}}$ for a single-point measurement. Additionally, static noise and autofocus repeatability were determined to be $2 \mathrm{~nm}$ and $5 \mathrm{~nm}$, respectively. The next phase of this research is to determine the other MCs from ISO/FDIS 25178-600 to allow uncertainty statements to be estimated with topography measurements. 


\section{ACKNOWLEDGEMENTS}

The authors would like to thank EPSRC (Grant EP/M008983/1) for funding this work.

\section{REFERENCES}

[1] Bruzzone A A G, Costa H L, Lonardo P M, Lucca D A 2008 Advances in engineered surfaces for functional performance CIRP Ann. - Manuf. Technol. 57:750-769.

[2] Thomas T R 2014 Roughness and function Surf. Topogr. Metrol. Prop. 2:14001.

[3] Ryk G, Etsion I 2006 Testing piston rings with partial laser surface texturing for friction reduction Wear 261:792-796.

[4] Etsion I, Sher E 2009 Improving fuel efficiency with laser surface textured piston rings Tribol. Int. 42:542-547.

[5] Etsion I, Halperin G, Becker E 2006 The effect of various surface treatments on piston pin scuffing resistance Wear 261:785-791.

[6] Bewilogua K, Bräuer G, Dietz A, Gäbler J, Goch G, Karpuschewski B, et al. 2009 Surface technology for automotive engineering CIRP Ann. - Manuf. Technol. 58:608-627.

[7] Ramsden J J, Allen D M, Stephenson D J, Alcock J R, Peggs G N, Fuller G, et al. 2007 The Design and Manufacture of Biomedical Surfaces CIRP Ann. - Manuf. Technol. 56:687-711.

[8] Evans C J, Bryan J B 1999 'Structured', 'textured' or `engineered' surfaces CIRP Ann. - Manuf. Technol. 48:541-556.

[9] Leach R K, Giusca C L, Haitjema H, Evans C, Jiang X 2015 Calibration and verification of areal surface texture measuring instruments CIRP Ann. - Manuf. Technol. 64:797-813.

[10] Giusca C L, Leach R K, Helary F, Gutauskas T, Nimishakavi L 2012 Calibration of the scales of areal surface topography measuring instruments: part 1. Measurement noise and residual flatness Meas. Sci. Technol. 23:65005.

[11] Giusca C L, Leach R K 2013 Calibration of the scales of areal surface topography measuring instruments: part 3. Resolution Meas. Sci. Technol. 24:105010.

[12] Giusca C L, Leach R K 2013 Calibration of the scales of areal surface topography measuring instruments : part 2 . Amplification, linearity and squareness Meas. Sci. Technol. 24:105010.

[13] Giusca C L, Claverley J D, Sun W, Leach R K, Helmli F, Chavigner M P J 2014 Practical estimation of measurement noise and flatness deviation on focus variation microscopes CIRP Ann. - Manuf. Technol. 63:545-548.

[14] Leach R K 2011 Optical Measurement of Surface Topography (Springer: Berlin).

[15] ISO 2014 25178-605 Geometrical product specifications (GPS) - Surface texture : Areal Part 605 : Nominal characteristics of non-contact (point autofocus probe) instrument.

[16] ISO 2016 25178-600 Geometrical product specification (GPS) - Surface texture: Areal Part 600: Metrological characteristics for areal-topography measuring methods.

[17] Haitjema H, Morel M A A 2005 Noise bias removal in profile measurements Meas. J. Int. Meas. Confed. 38:21-29.

[18] Leach R, Giusca C L, Rubert P A single set of material measures for the calibration of areal surface topography measuring instruments : the NPL Areal Bento Box. Met Props, p. 406-413.

[19] Maculotti G Determination of Metrological Characteristics for a Point Autofocus Instrument. 2017.

[20] de Groot P 2017 The meaning and measure of vertical resolution in optical surface topography measurement Appl. Sci. 7:1-6.

[21] ISO 2010 25178-701 Geometrical product specifications (GPS) - Surface texture : Areal Part 701 : Calibration and measurement standards for contact (stylus) instruments.

[22] Takamasu K 2011 Present problems in coordinate metrology for nano and micro scale measurements Mapan - J. Metrol. Soc. India 26:3-14.

[23] Garrat J D 1979 Survey of displacement transducers below $50 \mathrm{~mm}$ J. Phys. E. 241.

[24] Martin J, Tritt T, Uher C 2010 High temperature Seebeck coefficient metrology J. Appl. Phys. 
108: 121101.

[25] Haitjema H, Schellekens P H J, Wetzels S F C L 2000 Calibration of displacement sensors up to 300 um with nanometre accuracy and direct traceability to a primary standard of length Metrologia 37:25-33. 